\title{
Recovery in early stage breast cancer - An Australian longitudinal study
}

Karen-leigh EDWARD PhD, RN, GDipPsychology

Professor of Nursing and Practic-based Research| Swinburne University of Technology

Director of Research, St Vincent's Private Hospital Melbourne

Professor (visiting), School of Human and Health Science| University of Huddersfield, HD1 3DH| United Kingdom

59-61 Victoria Pde, Fitzroy VIC 3065 Australia

E: Karen-leigh.edward@svha.org.au

T: +61394117338

ORCID \# 0000-0001-8697-4006

Mitchell CHIPMAN MBBS FRACP

Medical Oncologist

Victorian Breast and Oncology Care

Level 2, 166 Gipps St

East Melbourne Vic 3002

E: Mitchell@ vboc.com.au

T: +61394174666

Dr John STEPHENSON PhD

Senior Lecturer in Biomedical Statistics

School of Human and Health Sciences | University of Huddersfield

Queensgate |Huddersfield HD1 3DH | United Kingdom

T: $+44(0) 1484471513$

E: J.Stephenson@hud.ac.uk

\section{Kayte ROBINSON RN}

Clinical Research and Data Manager

Victorian Breast and Oncology Care

Level 2, 166 Gipps St,

East Melbourne Vic 3002

E: Kayte@ vboc.com.au

T: +61394174666

Jo-Ann GIANDINOTO RN, BN (Hons), BBSc

PhD student

Australian Catholic University

E: jo-ann.giandinoto@ acu.edu.au

Roth TRISNO MBBS (Hons)

Medical Intern

Albury Wodonga Health

Vermont Street, Wodonga VIC 3690

PO Box 326, Albury NSW 2640

E: rtrisno@hotmail.com

T: +61 430555447

Conflicts of Interest - The authors do not have any conflicts of interest to declare.

Funding - This research was funded by the Australian College of Nurses. 


\section{ABSTRACT}

Background: The majority of breast cancer patients will experience some level of emotional distress, with some patients having long term psychological maladjustment. Personal and social resources play a role in recovery yet the interplay between these factors warrants further examination. This study aimed to investigate the interaction of psychosocial factors impacting women in their breast cancer trajectory, at two years or less following diagnosis (stages I-III).

Design: A longitudinal cohort study approach was used in this study.

Methods: The sample consisted of $n=49$ participants. Data were collected between June 2013 and October 2013 and followed for 12 months across the trajectory of the disease.

Results: The mean age was 56.6 years (SD 11.6 years). Most participants had stage I or stage II breast cancer. Time (over three time points -4 weeks, 6month and 12 months) after diagnosis was significantly associated with the body image $(p=0.003)$ and age $(p=0.004)$.

Conclusion: Older women with breast cancer reported less concern regarding body image than their younger peers. These findings suggest that post treatment younger women may require access to psychological support post treatment.

Key words: Breast cancer; Quality of life; Health-related quality of life; Body image; Psychological adjustment, Recovery trajectory 


\section{What is already known about this topic?}

- The majority of breast cancer patients experience some level of emotional distress, with some patients having long term psychological maladjustment.

- Personal resources (i.e. self-image, optimism, and perceived control) and social resources (i.e. social support) play a central role in recovery.

- The interplay between the personal and social resources is not well known particularly in the first two years post diagnosis

\section{What this paper adds?}

- Older women with breast cancer reported less concern regarding body image than their younger peers.

- Older women also experienced more positive future perspectives when compared to younger women.

- Younger women appeared less adaptive psychologically.

The implications of this paper?

- There are known psychological impacts for women who have breast cancer, and nurses are well placed within their scope of practice to undertake routine mental state examination of these patients.

- Younger women who have breast cancer in particular may require additional psychological supports during the first two years post diagnosis.

\section{INTRODUCTION}


Breast cancer is the most common cancer diagnosed in women in the developed and the developing world, comprising $25 \%$ of all cancers affecting women (Stewart W.B, 2014). When compared to all other cancers, breast cancer is the second most common cancer and due to its promising prognosis, is ranked fifth in cause of mortality (Ferlay et al., 2013). The many treatments for breast cancer offer women hope of survival where women have a greater chance for longer-term survival following diagnosis and treatment. Breast-conserving surgery, sentinel node biopsy, axillary dissection, mastectomy, major reconstructive surgery, radiotherapy, chemotherapy, hormonal therapy, antibody therapy, trials treatment with experimental drugs or a combination are examples of treatments offered to women (DeSantis et al., 2014). A diagnosis and the subsequent treatment of breast cancer, for example a surgical procedure and follow up drug treatment (often with adverse side effects), may introduce trauma and uncertainty for women (Pieters, 2016).

\section{Literature Review}

The majority of breast cancer patients will experience some level of emotional distress, with some patients having long term psychological maladjustment. Associated factors that impact upon psychological distress include disease-related variables, type of treatment and stage of disease (Markovitz, Schrooten, Arntz, \& Peters, 2015). Often individuals must locate their inner strength and use internal resources, such as being psychologically resilient, to cope with their individual illness course (Molina et al., 2014).

Diagnosis-related depression and anxiety can impact the recovery trajectory (Ng et al., 2017) . An early study by Weisman and Worden (1977), described the 'existential plight' of cancer recovery and suggests that in the first 100 days (two to three months) following diagnosis and treatment for cancer, patients experience their highest levels of emotional distress. Despite this early research there still remains limited longitudinal research focusing on the recovery trajectory for women with breast cancer. Personal resources (i.e. self-image, optimism, and perceived control) and social 
resources (i.e. social support) play a central role in recovery, yet the interplay between these factors warrants further examination, particularly in the first two years post diagnosis (Deimling, Kahana, \& Bowman, 2013; Ganz, Bower, \& Stanton, 2015).

\section{Study Aim}

This study aimed to investigate the interaction of psychosocial factors impacting women in their breast cancer trajectory, at two years or less following diagnosis (stages I-III). We aimed to investigate individual differences in regards to age, stage of cancer and psychosocial factors, such as body image, mood, bonding, satisfaction with life and resilience. The findings of this study were intended to inform the development of appropriate psychosocial interventions for women with breast cancer in the first two years post diagnosis.

\section{METHODS}

Design

A longitudinal cohort study approach was used in this pilot study.

\section{Sample/Participants}

Participants with a diagnosis of stages I, II or III breast cancer (diagnosis less than two years) were recruited to the study between June 2013 and October 2013 and followed for 12 months across the trajectory of the disease. Due to the longitudinal nature of the design data collection was completed in October 2015. As the study was considered to be a pilot, no formal sample size calculation was undertaken, however we aimed to recruit a convenience sample of 50 participants. The term 'older women' is used in reference the mean age of the sample. The setting for recruitment was a breast and oncology centre located in metropolitan Melbourne, Australia. 
A series of questionnaires were administered to patients at baseline (1-4 weeks post-operatively), at 6 months post-operatively and 12 months post-operatively. The outcomes of interest were health-related quality of life (HRQoL), partner support (intimate bonding), and wellbeing, satisfaction with life, resilience and mood. Data were collected using a survey approach and a researcher who was a breast care nurses engaged for the purposes of data collection recruited and collected data for the study at each time point.

Instruments and validity, reliability and rigour

HRQoL was assessed using the European Organisation for Research and Treatment of Cancer Quality of Life Breast Cancer Specific Version (EORTC QLQ-BR23) (Sprangers et al., 1996). It is a self-report tool which will take approximately 10 minutes to complete. The EORTC QLQ-BR23 has discriminant validity of mutually exclusive groups based on their initial performance status scores produced medium to large effect sizes ranging from 0.43 to 1.1 . Reliabilities ranged from 0.70 to 0.91 .

The Intimate Bond Measure (IBM) was used to measure partner support (i.e. perceptions of feeling controlled by their partner and feeling cared for by their partner), its properties have been assessed in separate studies, establishing its high test-retest reliability (Wilhelm \& Parker, 1988). The Intimate Bond Measure (IBM) is a self-report measure assessing two key underlying dimensions, care and control. Support for its validity, in terms of both perceived and actual characteristics of care and control, is demonstrated (Wilhelm \& Parker, 1988).

Subjective wellbeing was measured using the Satisfaction with Life Scale (SWLS), a short 5-item instrument designed to measure global cognitive judgments of satisfaction with one's life and has shown strong internal reliability and moderate temporal stability with a reliability coefficient $\alpha$ of 0.87 and a 2-month test-re-test stability coefficient of 0.82 (Diener, Emmons, Larsen, \& Griffin, 1985). 
Resilience was measured using the Connor-Davidson Resilience Scale (CD-RISC), it has been tested in the general population, as well as in clinical samples, and demonstrates excellent psychometric properties, with good internal reliability and test-retest reliability (Connor \& Davidson, 2003) and a Cronbach's alpha $=0.91$.

Depression, anxiety or stress was measured using the Depression Anxiety Stress Scales (DASS). The scale consists of a total of 42 items with a 0-3 Likert scale and Cronbach's $\alpha$ statistic for internal reliability, is 0.91 for the Depression subscale, 0.84 for the Anxiety subscale and 0.90 for the Stress subscale (Antony, Bieling, Cox, Enns, \& Swinson, 1998). High scores on each subscale of this instrument are associated with higher levels of depression, anxiety and stress. For this study the DASS (21) was used. It has the advantage of taking half the time to complete, reducing participant burden. This version of the scale has 21 items (7 items for each emotional state).

Demographic data including age and the stage of cancer at recruitment were recorded, classified as stage I, II or III.

\section{Ethical statement}

This study was conducted in accordance with the Declaration of Helsinki, ethical principles for medical research involving humans. Ethical approval was received from the University Human Research Ethics Committee [2013 -147V] and the hospital Human Research Ethics Committee [LRR: 098/13]. Due to the nature of the condition under investigation, all patients recruited to the study were female. Informed consent was obtained from all individual participants prior to their inclusion in the study. Potential distress was managed with referral back to the treating oncologist or to the person's general practitioner. Also, support services such as Lifeline were identified to participants as options for additional support should they require it. 
Repeated measures analyses of variance (ANOVAs) were conducted on each scale/subscale. Subscales associated with the same scale were analysed using doubly multivariate repeated measures ANOVAs. All analyses controlled for stage of cancer and patient age. All scales and subscales were analysed based on changes between baseline and 12 months, which was considered to be the primary analysis period. Secondary analyses based on changes between baseline and 6 months, and between 6 months and 12 months, were also conducted on any scale or subscale for which inspection of profiles indicated substantial deviations from a linear trend. To avoid extensive casewise deletion of data, the extent of missing data on each measured subscale was assessed, with any scale with a substantial proportion of missing data being analysed separately from the analysis of other subscales. All analyses were conducted using International Business Machines Corporation SPSS statistical software (Version 22.0) (SPSS, 2011).

\section{RESULTS}

\section{Descriptive analysis}

Data were originally obtained from 52 participants. One participant did not complete any of the questionnaires and was removed from the analysis. All other participants completed all questionnaires at baseline and were included in subsequent analyses, which were hence based on a sample size of $n=51$. One participant failed to complete questionnaires at the 6 -month data collection time point, but subsequently provided valid data at the 12 -month data collection time point. One participant withdrew from the study between the 6-month and 12-month data collection time points. Hence a full set of follow-up data was received from $n=49$ patients $(96.1 \%$ of those providing valid data).

In most case patients' surgery involved either mastectomy or lumpectomy. Some patients' treatment also included adjunctive therapies such as chemotherapy and radiotherapy. Detailed 
information about individual surgical data were not collected. The mean age of participants at baseline was 56.6 years (SD 11.6 years). Most participants were married with children, and were Australian-born native English speakers. Slightly less than half had a university education. Around half of the participants had a family history of cancer and most did not report any co-morbidities. Most participants had stage I or stage II breast cancer.

All categorical health and demographic factors are summarised in Table 1 below.

[insert table 1 here]

Due to the low frequency of participants with stage III cancer, these patients were combined with those with stage II cancer, for a subsequent comparative analysis of stage I cancer versus stage II or stage III cancer. Means and standard deviations of scores obtained on all subscales of all questionnaires at baseline, 6 months and 12 months; plus the number of valid responses in each case, are given in Table 2 below. All reported scores are transformed from the raw scores following the procedures appropriate to each questionnaire.

[insert table 2 here]

A small proportion of questions on all three questionnaires were not completed or recorded as not applicable. The low frequencies of responses to the sexual enjoyment subscale, which was conditional on responses to the sexual functioning subscale, led to the requirement that the sexual enjoyment subscale be analysed independently of other BR23 QoL functional subscales, to avoid extensive loss of information from cases with missing sexual enjoyment data. The very low frequency of responses to the upset by hair loss subscale at baseline, which was also conditional on responses to other questionnaire items, led to this subscale being deleted from further analysis. 
Primary analyses: Repeated measures analyses of variance

Doubly multivariate repeated measures ANOVAs controlling for age and stage of cancer revealed that the time after diagnosis was significantly associated with the Body image and a significant interaction between age and time after diagnosis indicating that the effect of time was not the same for individuals of different ages. All significant and substantive associations revealed by the ANOVA models are summarised in Table 3.

[insert table 3 here]

Secondary analyses: Depression subscale of DASS scale scores

Following the findings of the descriptive analyses, repeated measures ANOVAs were conducted on the Depression subscale of the DASS scale using baseline data and data collected at 6 months; and using data collected at 6 months and at 12 months. Both analyses were controlled for age and stage of cancer. The most substantial trend is observed in the systemic therapy side effects subscale scores of the $B R 23$ QoL scale. However, following deterioration in symptoms between baseline and 6 months, after 12 months this measure returned to about its baseline level. The same pattern is observed in all three subscales of the DASS scale (depression, anxiety and stress scale).

\section{DISCUSSION}

Depression is common in women receiving therapy for the treatment of breast cancer (Reece, Chan, Herbert, Gralow, \& Fann, 2013) and some treatment side effects may exacerbate mood disturbance due to other neurocognitive changes commonly experienced as a result of cancer therapies (Dietrich \& Parsons, 2018; Winocur, Johnston, \& Castel, 2018). Women with breast cancer are at long-term increased risk for depression, including both severe episodes leading to hospitalisation and use of antidepressants (Suppli et al., 2014). In addition women may experience distress due to a poor body 
image and /or depression due to treatment effects, such as chemotherapy-induced alopecia (Choi et al., 2014).

The greatest contrast between baseline and 12-month values was body image. However, the deterioration in body image takes place mainly over the first 6 months, but unlike the other measures does not recover over the subsequent 6 months. Body image was found to be better in older women than younger women at both time points and this gap widened over the 12 month period. Treatment that involves the removal of breasts is known to impact on a woman's psychological wellbeing including body image and can negatively affect the woman's body image and personal resilience (Henderson et al., 2012; Hughes, Edward, \& Hyett, 2011). The type of intervention (i.e. breast conserving surgery versus mastectomy or mastectomy and upfront reconstruction) may have additional age related impacts (Somogyi et al., 2015). In addition, other treatment effects for example the effects of lymphedema can also impact body image (Rosenberg et al., 2013). Importantly there is growing evidence that younger women are at increased risk of poorer quality of life with regards to body image than their older counterparts potentially due to the presence of menopausal symptoms coupled with a breast cancer diagnosis which can lead to impaired sexual functioning; and in young women this loss of sexual functioning can lead to depression (Siegel et al., 2012).

Having a positive future outlook was seen to be better in older women when compared to younger women at both time points in our study. Other research suggests younger cancer survivors reported higher levels of psychological distress, and this was often attributed to uncertainty for the future (Hall, Mishel, \& Germino, 2014) and potentially poor psychological adaptation. Some authors suggest optimism does not moderate the causal relationship between fear of cancer returning and 
psychological wellbeing (Dumalaon-Canaria, Prichard, Hutchinson, \& Wilson, 2016) while other researchers suggest optimism does lead to better mental and physical functioning including better wellbeing (Colby \& Shifren, 2013). Nevertheless, to foster psychological adaptation or resilience, positive interventions applied to patients and survivors of breast cancer promotes positivity (Casellas-Grau, Font, \& Vives, 2014).

\section{Study Limitations}

This study's findings have provided good pilot data for a larger study examining age related impacts of breast cancer in more detail. The small size $(n=49)$ of this pilot study precluded attainment of sufficient power to detect significant effects. Despite this, several inferences of significance have been made, suggesting a fully-powered full-scale study would be expected to show at least some significant findings. The lack of significance of stage of cancer as a main effect with respect to any outcome measure may be directly related to the low power of the study; or it may be a consequence of the low numbers of stage III cancer patients. The combination of stage II and stage III cancer patients into a single group, the majority of which were stage II patients, led to analysis which essentially compared stage I against stage II; however, the development of cancer and its consequences might be expected to be more profound between stages II and III than between stages I and II. The current investigation has utilised repeated measures analysis of variance and doubly multivariate repeated measures analysis of variance models without corrections for multiple comparisons. The retention rate of the study of over $96 \%$ was very high for a longitudinal study of this kind and minimises the risk of attrition bias.

\section{CONCLUSION}

Age-related impacts were noted in the course of recovery for women who have a diagnosis (and treatment) of breast cancer $<2$ years post diagnosis and initial treatment. Older women with breast 
cancer appeared to do better than their younger peers in relation to having a better body image, where the gap observed between younger and older women with regard to body image appeared to widen over time. Older women also experienced more positive future perspectives when compared to younger women. It is possible that this can be attributed to stage of life expectations or life experience knowledge assisting older women to psychologically adapt to their health state. Younger women appeared less adaptive psychologically, therefore individualised age-related interventions in the first 6 months post-diagnosis is recommended especially for younger women.

\section{Clinical Implications}

Nurses and other healthcare professionals need to facilitate the individualised assessment of potential psychological supports that women may require, such as using the mental state examination as part of a routine nursing assessment in oncology. In addition, contemporary healthcare practices are moving towards identifying and working with patients' strengths including their protective factors (i.e. personal resilience) as a means of empowering patients in their illness trajectory, facilitating the maintenance of emotional stability and while this is commonly seen in mental health settings (Rani, 2015) acute medical care services are yet to follow this lead (Edward, 2013). Higher levels of resilience are associated with better emotional adjustment in breast cancer patients (Markovitz et al., 2015) and in light of this last point, working with breast cancer survivors strengths could be integrated into routine clinical care interventions potentially offering a means to mitigate the related impacts. 


\section{REFERENCES}

Antony, M. M., Bieling, P. J., Cox, B. J., Enns, M. W., \& Swinson, R. P. (1998). Psychometric properties of the 42-item and 21-item versions of the Depression Anxiety Stress Scales in clinical groups and a community sample. Psychological Assessment, 10(2), 176.

Casellas-Grau, A., Font, A., \& Vives, J. (2014). Positive psychology interventions in breast cancer. A systematic review. Psycho-Oncology, 23(1), 9-19.

Choi, E. K., Kim, I. R., Chang, O., Kang, D., Nam, S. J., Lee, J. E., .. Y Yang, J. H. (2014). Impact of chemotherapyinduced alopecia distress on body image, psychosocial well-being, and depression in breast cancer patients. Psycho-Oncology, 23(10), 1103-1110.

Colby, D. A., \& Shifren, K. (2013). Optimism, mental health, and quality of life: a study among breast cancer patients. Psychology, health \& medicine, 18(1), 10-20.

Connor, K. M., \& Davidson, J. R. (2003). Development of a new resilience scale: The Connor-Davidson resilience scale (CD-RISC). Depression And Anxiety, 18(2), 76-82.

Deimling, G. T., Kahana, B., \& Bowman, K. (2013). Adaptation and Adjustment to Cancer in Later Life: A Conceptual Model. Cancer and Aging Handbook: Research and Practice, 365-383.

DeSantis, C. E., Lin, C. C., Mariotto, A. B., Siegel, R. L., Stein, K. D., Kramer, J. L., . . Jemal, A. (2014). Cancer treatment and survivorship statistics, 2014. CA: A Cancer Journal for Clinicians, 64(4), 252-271. doi:doi:10.3322/caac.21235

Diener, E., Emmons, R. A., Larsen, R. J., \& Griffin, S. (1985). The satisfaction with life scale. Journal of Personality Assessment, 49(1), 71-75. doi:10.1207/s15327752jpa4901_13

Dietrich, J., \& Parsons, M. W. (2018). Cognitive Dysfunction, Mood Disorders, and Fatigue as Complications of Cancer Cancer Neurology in Clinical Practice (pp. 203-219): Springer.

Dumalaon-Canaria, J. A., Prichard, I., Hutchinson, A. D., \& Wilson, C. (2016). Fear of cancer recurrence and psychological well-being in women with breast cancer: The role of causal cancer attributions and optimism. European Journal of Cancer Care, 10/2016, 1-12. doi:10.1111/ecc.12579

Edward, K.-I. (2013). Chronic illness and wellbeing: using nursing practice to foster resilience as resistance. British Journal of Nursing, 22(13), 741-746.

Ferlay, J., Steliarova-Foucher, E., Lortet-Tieulent, J., Rosso, S., Coebergh, J., Comber, H., . . Bray, F. (2013). Cancer incidence and mortality patterns in Europe: estimates for 40 countries in 2012. European Journal of Cancer, 49(6), 1374-1403.

Ganz, P. A., Bower, J. E., \& Stanton, A. L. (2015). Special issues in younger women with breast cancer Improving Outcomes for Breast Cancer Survivors (pp. 9-21): Springer.

Hall, D. L., Mishel, M. H., \& Germino, B. B. (2014). Living with cancer-related uncertainty: associations with fatigue, insomnia, and affect in younger breast cancer survivors. Supportive Care in Cancer, 22(9), 2489-2495.

Henderson, V. P., Clemow, L., Massion, A. O., Hurley, T. G., Druker, S., \& Hebert, J. R. (2012). The effects of mindfulness-based stress reduction on psychosocial outcomes and quality of life in early-stage breast cancer patients: A randomized trial. Breast cancer research and treatment, 131(1), 99-109.

Hughes, S., Edward, K., \& Hyett, B. (2011). Enhancing nursing practice potential: breast free flap reconstruction following mastectomy. Australian Nursing Journal, 19(2), 30-34.

Markovitz, S. E., Schrooten, W., Arntz, A., \& Peters, M. L. (2015). Resilience as a predictor for emotional response to the diagnosis and surgery in breast cancer patients. Psycho-Oncology, 24(12), 16391645.

Molina, Y., Yi, J. C., Martinez-Gutierrez, J., Reding, K. W., Yi-Frazier, J. P., \& Rosenberg, A. R. (2014). Resilience among patients across the cancer continuum: diverse perspectives.(Report). Clinical Journal of Oncology Nurses, 18(1), 93-101. doi:10.1188/14.CJON.93-101

Ng, C. G., Mohamed, S., Kaur, K., Sulaiman, A. H., Zainal, N. Z., Taib, N. A., \& group, M. S. (2017). Perceived distress and its association with depression and anxiety in breast cancer patients. PloS one, 12(3), e0172975.

Pieters, H. C. (2016). "I'm Still Here": Resilience Among Older Survivors of Breast Cancer. Cancer Nursing, 39(1), E20-E28. 
Rani, U. (2015). The strengths model: A recovery-oriented approach to management of people suffering with severe mental illness. Indian Journal of Health and Wellbeing, 6(4), 445.

Reece, J. C., Chan, Y.-F., Herbert, J., Gralow, J., \& Fann, J. R. (2013). Course of depression, mental health service utilization and treatment preferences in women receiving chemotherapy for breast cancer. General hospital psychiatry, 35(4), 376-381.

Rosenberg, S. M., Tamimi, R. M., Gelber, S., Ruddy, K. J., Kereakoglow, S., Borges, V. F., . . Partridge, A. H. (2013). Body image in recently diagnosed young women with early breast cancer. Psycho-Oncology, 22(8), 1849-1855.

Siegel, R., DeSantis, C., Virgo, K., Stein, K., Mariotto, A., Smith, T., ... Fedewa, S. (2012). Cancer treatment and survivorship statistics, 2012. CA: A Cancer Journal for Clinicians, 62(4), 220-241.

Somogyi, R. B., Webb, A., Baghdikian, N., Stephenson, J., Edward, K.-I., \& Morrison, W. (2015). Understanding the factors that influence breast reconstruction decision making in Australian women. The Breast, 24(2), 124-130.

Sprangers, M., Groenvold, M., Arraras, J. I., Franklin, J., te Velde, A., Muller, M., . . Hopwood, P. (1996). The European Organization for Research and Treatment of Cancer breast cancer-specific quality-of-life questionnaire module: first results from a three-country field study. Journal of Clinical Oncology, 14(10), 2756-2768.

SPSS, I. (2011). SPSS Statistics 20. SPSS Inc, Chicago, IL.

Stewart W.B, a. (2014). World Cancer Report 2014: Geneva : IARC.

Suppli, N. P., Johansen, C., Christensen, J., Kessing, L. V., Kroman, N., \& Dalton, S. O. (2014). Increased risk for depression after breast cancer: A nationwide population-based cohort study of associated factors in Denmark, 1998-2011. Journal of Clinical Oncology, 32(34), 3831-3839.

Weisman, A. D., \& Worden, J. W. (1977). The existential plight in cancer: Significance of the first 100 days. The International Journal of Psychiatry in Medicine, 7(1), 1-15.

Wilhelm, K., \& Parker, G. (1988). The development of a measure of intimate bonds. Psychological Medicine, $18(1), 225-234$.

Winocur, G., Johnston, I., \& Castel, H. (2018). Chemotherapy and cognition: international cognition and cancer task force recommendations for harmonising preclinical research. Cancer treatment reviews. 
Table 1: Descriptive summary of participants

\begin{tabular}{|c|c|}
\hline Variable & Frequency (\%) \\
\hline \multicolumn{2}{|l|}{ Family status } \\
\hline Married or living with partner & $39(76.5 \%)$ \\
\hline Living with other family member(s) & $8(15.7 \%)$ \\
\hline Single & $3(5.9 \%)$ \\
\hline \multicolumn{2}{|l|}{ Children } \\
\hline No children & $10(19.6 \%)$ \\
\hline One or more children & $41(80.4 \%)$ \\
\hline \multicolumn{2}{|l|}{ Language spoken } \\
\hline English & $49(96.1 \%)$ \\
\hline Other/not answered & $2(3.9 \%)$ \\
\hline \multicolumn{2}{|l|}{ Country of birth } \\
\hline Australia & $38(74.5 \%)$ \\
\hline Other/no answer & $13(25.5 \%)$ \\
\hline \multicolumn{2}{|l|}{ Highest level of education } \\
\hline High school & $13(25.5 \%)$ \\
\hline Tertiary education & $10(19.6 \%)$ \\
\hline University (Bachelor's degree or above) & $22(43.1 \%)$ \\
\hline Other/declined to respond & $6(11.8 \%)$ \\
\hline \multicolumn{2}{|l|}{ Co-morbidities } \\
\hline Yes & $22(43.1 \%)$ \\
\hline No/not stated & $29(56.9 \%)$ \\
\hline \multicolumn{2}{|l|}{ Family history of cancer } \\
\hline Yes & $25(49.0 \%)$ \\
\hline No & $26(51.0 \%)$ \\
\hline \multicolumn{2}{|l|}{ Stage of cancer at entry to study } \\
\hline Stage I & $22(43.1 \%)$ \\
\hline Stage II & $21(41.2 \%)$ \\
\hline Stage III & $8(15.7 \%)$ \\
\hline
\end{tabular}

Table 2: Summary of questionnaire responses (mean (SD))

\begin{tabular}{|lllll|}
\hline Scale & Subscale & Baseline & 6 months & 12 months \\
IBM & Care & $28.6(7.49) ; n=43$ & $24.9(9.94) ; n=42$ & $26.3(8.95) ; n=43$ \\
& Control & $4.74(5.07) ; n=43$ & $5.55(4.99) ; n=42$ & $5.47(4.52) ; n=43$ \\
CD-RISC & - & $76.8(15.2) ; n=51$ & $72.8(15.7) ; n=50$ & $72.8(14.6) ; n=50$ \\
SWLS & - & $26.9(6.97) ; n=51$ & $24.3(7.66) ; n=50$ & $26.4(6.70) ; n=50$ \\
BR23 & Future perspective & $46.7(31.3) ; n=50$ & $49.7(31.7) ; n=49$ & $52.8(31.8) ; n=49$ \\
& Body image & $85.0(20.7) ; n=50$ & $75.9(24.6) ; n=49$ & $77.4(26.6) ; n=48$ \\
& Sexual functioning & $21.2(21.4) ; n=49$ & $21.1(20.7) ; n=49$ & $25.7(25.7) ; n=48$ \\
& Sexual enjoyment & $58.3(27.6) ; n=20$ & $54.0(33.3) ; n=21$ & $57.7(28.6) ; n=26$ \\
BR23 & Systemic therapy side effects & $11.5(12.9) ; n=49$ & $27.6(24.1) ; n=49$ & $18.5(15.4) ; n=47$ \\
& Upset by hair loss & $41.7(36.3) ; n=4$ & $42.9(29.4) ; n=21$ & $39.6(31.7) ; n=16$ \\
& Arm symptoms & $15.7(18.7) ; n=51$ & $13.6(15.8) ; n=50$ & $11.8(17.0) ; n=49$ \\
& Breast symptoms & $23.4(17.8) ; n=51$ & $19.7(20.1) ; n=50$ & $14.8(15.2) ; n=50$ \\
DASS & Depression & $5.14(7.29) ; n=51$ & $8.48(8.84) ; n=50$ & $5.20(7.14) ; n=50$ \\
& Anxiety & $3.96(4.66) ; n=51$ & $6.04(7.05) ; n=50$ & $4.24(5.32) ; n=50$ \\
& Stress & $8.86(7.71) ; n=51$ & $10.4(9.62) ; n=50$ & $9.04(8.69) ; n=50$ \\
\hline
\end{tabular}

Key: BR23 (QoL Scale -symptom measures); DASS (Depression, Anxiety and Stress Scale); SWLS (Satisfaction with Life Scale); CD-RISC (Connor Davidson Resilience Scale); IBM (Intimate Bond Measure); SWLS (Satisfaction with Life Scale). * Significance is p<.05 
Table 3: Summary of associations for primary analysis period (0-12 months)

\begin{tabular}{|c|c|c|c|c|c|c|}
\hline Scale & Subscale & Variable & F-ratic & dff & $p$-value & $\begin{array}{l}\text { Effect size } \\
\left(\text { partial- } \eta^{2}\right)\end{array}$ \\
\hline IBM & Control & Age & 5.93 & 1,39 & $0.020 *$ & 0.132 \\
\hline CD-RISC & - & Time $\mathrm{x}$ stage & 3.59 & 1,47 & 0.064 & 0.071 \\
\hline \multirow[t]{4}{*}{ BR23 } & Future perspective & Age & 9.32 & 1,40 & $0.004 *$ & 0.189 \\
\hline & Body image & Time & 13.4 & 1,40 & $0.001 *$ & 0.250 \\
\hline & & Age & 12.0 & 1,40 & $0.001 *$ & 0.230 \\
\hline & & Age $x$ time & 10.2 & 1,40 & $0.003^{*}$ & 0.204 \\
\hline
\end{tabular}

